\title{
Radioactive Particles in Samples of PM10 by SEM-EDS
}

\author{
Roberto Ramirez-Leal ${ }^{1}$, Martin Cruz-Campas ${ }^{1}$, Daniel Morales-Romero ${ }^{2}$ and Octavio Cota-Arriola ${ }^{1}$ \\ ${ }^{1}$ SONORA STATE UNIVERSITY, hermosillo, Sonora, Mexico, ${ }^{2}$ Sonora State University, United States
}

In recent decades, great efforts have been focused on the study of atmospheric aerosol due to the fundamental role it plays in the chemical and physical processes that occur in the atmosphere, and these constitute one of the main means of transporting pollutants. Although at the beginning the studies did not detect important differences between the effects observed in different cities around the world, recent improvements in the methodologies used have made it possible to show significant variations in the effects observed between cities. [1,2]

More recent studies have incorporated other components of particulate matter (PM) into their analysis, which go beyond the standard mass criterion and include: organic and elemental carbon; some radioactive ions and elements, such as uranium and thorium. The results seem to confirm that the composition of the particles could explain the variability in the impact on health, depending on the characteristics of each city. The main source of radiation exposure to humans is that of natural radiation in the environment, these naturally occurring radionuclides are present at various levels in all soils, depending upon the local geology. Airborne radionuclides are adsorbed on the surface of aerosol particles and form radioactive aerosol. Therefore the behavior of the airborne radionuclide particles is determined by the behavior of their carrier aerosol particles. In this context, it is of fundamental importance to understand the origin (natural or anthropogenic) of particles in the atmosphere. [3]

The aim of this work is the identification and characterization (morphology and chemical composition) of particles that present radioactive elements in PM10 (particles less than 10 microns) samples as part of an air quality study by SEM-EDS.

The City of Hermosillo is located between latitude $20^{\circ} 01^{\prime} 00$ "and $20^{\circ} 08^{\prime} 30^{\prime \prime}$ north latitude and between the meridian $110^{\circ} 54^{\prime} 30$ "and $111^{\circ} 01^{\prime} 00^{\prime \prime}$ west longitude at an altitude of $200 \mathrm{~m}$ above sea level; is the Sonora state's capital, located to the Center-West of the coastal plain, in northwestern Mexico [3].

The PM10 particles was collected by high-volume sampler, located in three atmospheric monitoring stations of the Air Quality Improvement Municipal Program for the City of Hermosillo, Sonora, Mexico, using a sampling flow of $1.3 \mathrm{~m} 3 / \mathrm{min}$. Sampling was carried for 24 -h one day a week. The filters used were quartz, with size of $8 \times 10$ inch that captured PM10 particles. Analysis of PM10 were performed using scanning electron microscopy (SEM) JEOL Model JSM-5800LV coupled with energy dispersive spectrometer (EDS) EDAX DX prime brand, for determination of morphology and elemental composition of airborne particles.

Specimens were processed by separating the collected particles from the quartz filters by means of submersing a $2 \mathrm{~cm}^{2}$ section of each filter into isopropilic alcohol within a test tube for 5 minutes. Then, an aliquot of the suspension was placed over a sample holder, and is introduced into the chamber of SEM. In our study, it was observed that the uranium-containing particles present an irregular morphology, with peripheral edges and fracture lines. (Fig. 1) Based on the EDS results, the following elements were found: $\mathrm{Al}, \mathrm{Ca}, \mathrm{K}, \mathrm{Mg}, \mathrm{Na}, \mathrm{S}, \mathrm{Si}, \mathrm{U}$ and V; and the predominant elements were Si (At\% 8.23), V (At\% 2.12) and U (At\% 2.11). (Fig. 2). 
The results obtained from the characterization of the particles captured by PM10 filters, showed that elements such as $\mathrm{Al}, \mathrm{Ca}, \mathrm{K}, \mathrm{Mg}$ and $\mathrm{Si}$ are largely abundant, and are usually related with litophilic sources, areas of soil free of anthropogenic impact, paved streets and construction sites. $\mathrm{S}$ is an element that is typical of mobile emission sources. $U$ is a natural element in areas of high mining activity. Comparing the morphology and chemical composition of the uranium-containing particles, we obtain that the source is the natural one; and which are made up of oxides and carbonates of silica, calcium and uranium. The data indicate that $\mathrm{U}$ is more than likely an intrinsic component of the mineral matrix.
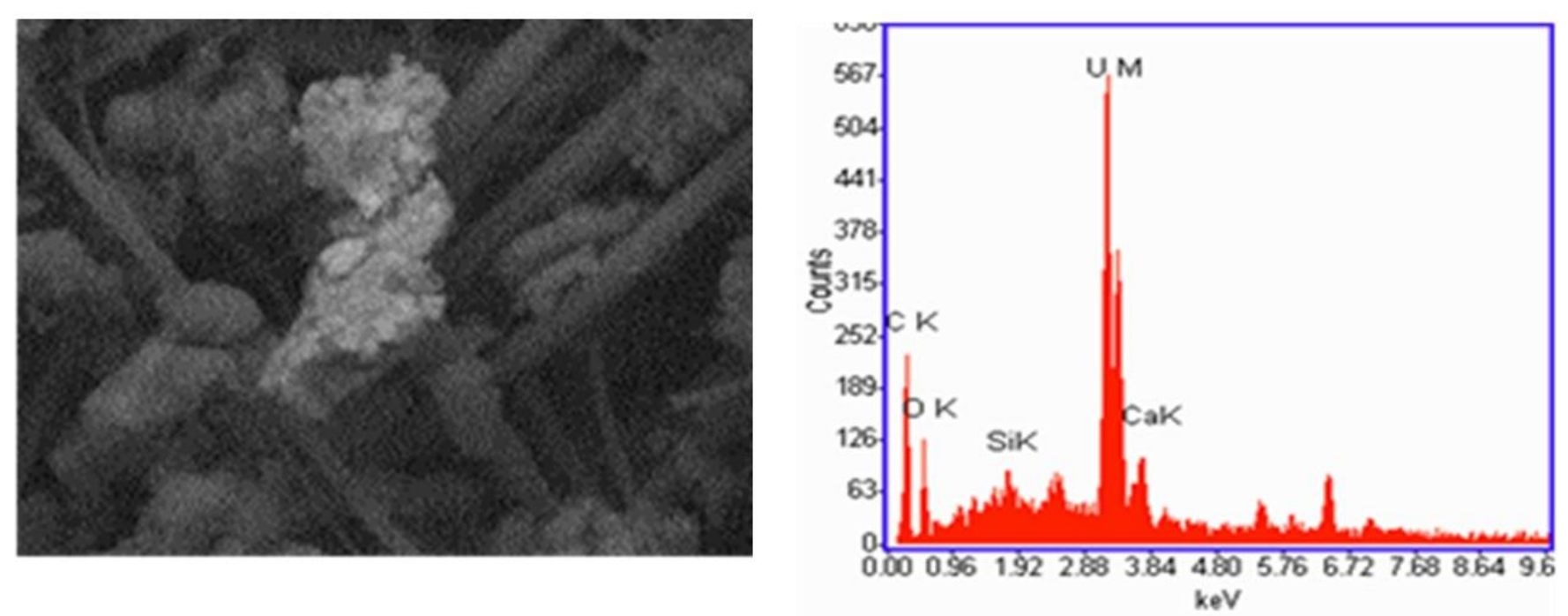

Figure 1. Micrograph of the sample in SEM-EDS. 6000x, BEI and $15 \mathrm{Kv}$

\section{References}

1.- Ramirez-Leal, R., Esparza-Ponce,H and Duarte-Moller,A. (2007). Characterization of inorganic atmospheric particles in air quality program with sem, tem and xas. Revista Mexicana de Fisica 53(3): 102-107.

2.- Ramirez-Leal, R.,Valle-Martinez,M. and Cruz-Campas, M. (2014). Chemical and Morphological Study of PM10 Analysed by SEM-EDS. Open Journal of Air Pollution, 3, 121-129

3.- M. Cruz-Campas, R. Ramirez-Leal and N. Lopez-Perez. (2019). TSP Analysis Performed by SEMEDS to Air Quality Studies. Microsc. Microanal. 25 (Suppl 2) 